\title{
Face Recognition using Independent Component Analysis Algorithm
}

\author{
Zaid Abdi Alkareem Alyasseri \\ ECE Dept. / Faculty of Engineering \\ University of Kufa/lraq
}

\begin{abstract}
Face recognition system is an intelligent application, which is able to identify or verify a person from digital sources such as (digital image or a video stream). In spite of the existence of alternative technologies such as fingerprint and iris recognition, the human face remains one of the most popular cues for identity recognition in biometrics. The easy way to recognize any face is by comparing the facial features from the input image (tested image) with a facial database. The most important task in face recognition is how to find the best matching between the tested and training faces. In this paper, how to recognize a face is presented; for evaluating the proposed system, the author was used two various analysis algorithms which are Eigenface and Independent Component Analysis (ICA). The local dataset used in this paper is preprocessed using statistical standard methods. Pre-processing software, Face Identification Evaluation System Version 5.0 under Unix Shell scripts, was written via ANSII C code, which is provided by the Colorado State University (CSU). Independent Component Analysis algorithm (ICA) is written using Matlab R2012b for face recognition implementation. Finally, the results show several graphs by Matlab. In addition, the proposed system shows the best results in Illumination images comparing with Occlusion images.
\end{abstract}

\section{General Terms}

Pattern Recognition, Image processing, ICA Algorithms

\section{Keywords}

Pattern Recognition, Face Recognition System, ICA algorithm, Eigenface

\section{INTRODUCTION}

During the past three decades, extensive research has been conducted on automatically recognizing the identity of individuals based on their facial images. In spite of the existence of alternative technologies such as fingerprint and iris recognition, the human face remains one of the most popular cues for identity recognition in biometrics. Face recognition possesses the non-intrusive nature and is often effective without the participant's cooperation or knowledge. It makes a good compromise between performance reliability and social acceptance and well balances security and privacy. Other biometric methods do not possess these advantages. For instance, fingerprint recognition methods require the subjects to cooperate in making explicit physical contact with the sensor surface [1]. Similarly, iris recognition methods require the subjects to cooperate in placing their eyes carefully relative to the camera. Nowadays, face recognition is one of the most successful applications of image analysis and understanding as well as face recognition has become the widest field for researchers in computer vision [2]. Face recognition system is a biometric technique that is used in law enforcement agencies, personal identification systems, and for security purposes. This computer application automatically identifies a person from a digital image [3]. There are several algorithm have been used for face recognition for instance PCA, AMM, LDA, SVM, EBGM,EP , HMM and ICA [2].

One of the most popular unsupervised statistical approaches that is used for finding the best method of image representations is Principal Component Analysis (PCA). Moreover, it was applied in the most successful representations algorithms for face recognition system such as local feature analysis [4], eigenfaces [5], and holons [6]. In a task such as face recognition, much of the important information may be contained in the high-order relationships among the image pixels, and, thus, it is important to investigate whether generalizations of the PCA, which are sensitive to high-order relationships, not just second-order relationships, are advantageous. Independent component analysis (ICA) [7] is one such generalization. An algorithm for performing ICA has been proposed. In this article, we do the task using ICA algorithm and eigenvectors. The approach transforms face images into a small set of characteristic feature images, called "eigenface," which are the principal component of the initial training set of face images [8]; then, we apply the Independent Component Analysis (ICA), which is a statistical and computational technique used for revealing hidden factors that underlie sets of random variables, measurements, or signals [9]. The first step for a face recognition system is to recognize a human face and extract it from the rest of the scene. Next, the system measures nodal points on the face, such as the eyes and mouth; these nodal points are used to remove the background of the image and keep the statistical feature (distinct face).

The rest of this paper is organized as follows. Section 2 provides the necessary face recognition related work. Section 3 describes enrollment images for the proposed system (3.1), preprocessing the local images dataset (3.2), and the image recognition system (3.3). Section 4 shows experimental results and discussion. Section 5 concludes with practical recommendations.

\section{RELATED WORK}

Through the past three decades the face recognition systems have been developed. Indeed, this development depending on three kinds of recognition algorithms, namely profile, frontal, and view tolerant recognition, based on the type of images and the recognition algorithms.

In this part, the author presents an overview of the major human face recognition techniques that apply mostly to frontal faces; the strengths and weaknesses of each approaches are also given. The methods considered are dynamic link architecture, neural networks, hidden Markov model, eigenfaces, geometrical feature matching, and template matching. The methods are analyzed in terms of the facial representations they use [10]. 


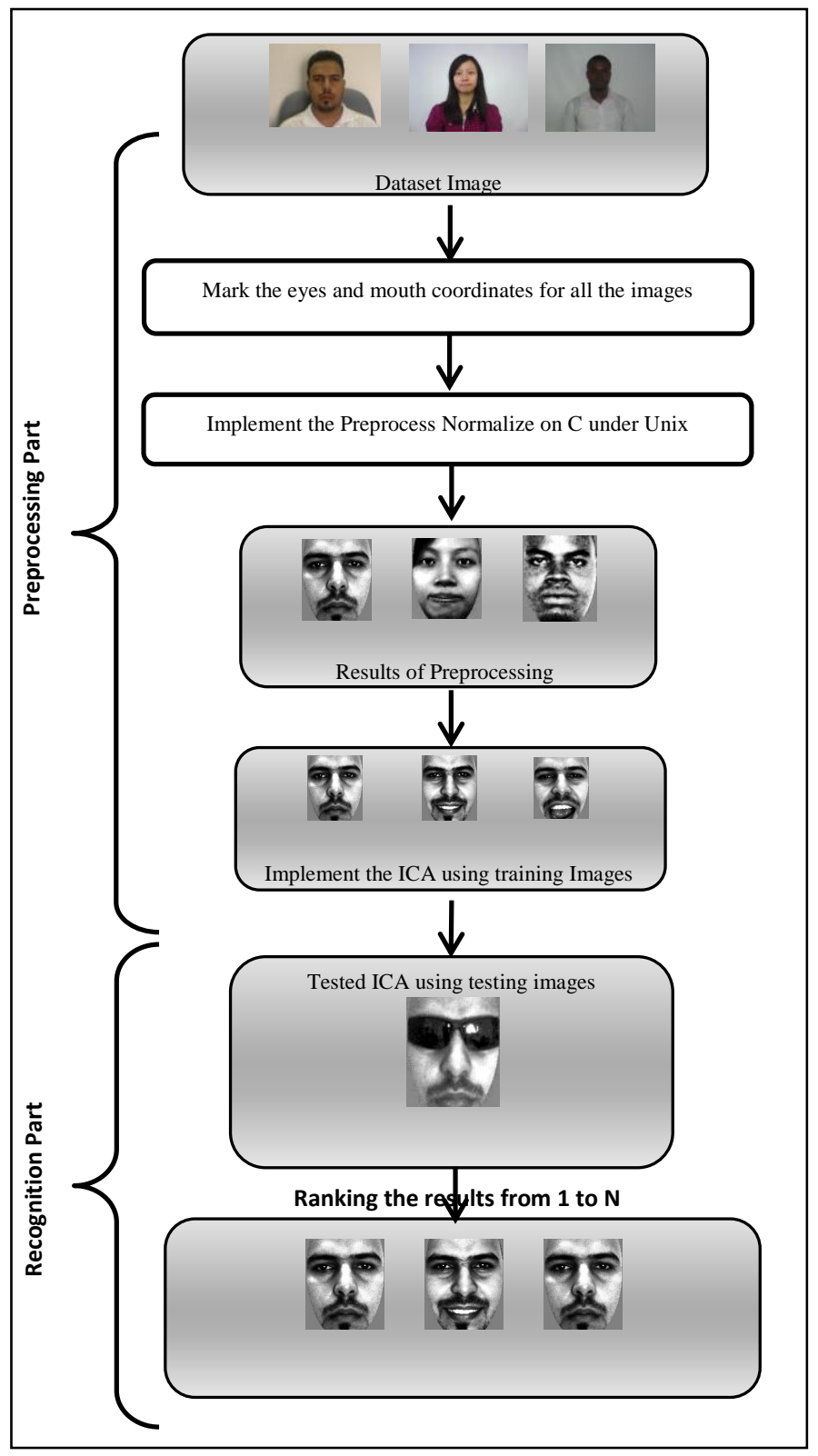

Figure 1 Overall system

Bell and Sejnowski $[11,12]$ developed an algorithm from the point of view of optimal information transfer by using neural networks with sigmoidal transfer functions. The proposed algorithm has proved successful for separating randomly mixed auditory electroencephalogram (EEG) signals and functional magnetic resonance imaging (fMRI) signals.

The performance of Independent Component Analysis (ICA) relies on the function, the algorithm used to approximate ICA, and the number of subspace dimensions retained. Actually, the procedure of apply Independent Component Analysis (ICA) algorithm for face recognition should have two different ways. At the first side, the proposed algorithm (ICA) can be used as to process images as random variables and pixels as observations, the second way, it can be used as to process pixels as random variables and images as observations. According to [13, 14], the authors described, two methods for ICA architecture I and II, respectively. Where ICA architecture I applied for localized tasks and ICA architecture II applied for holistic tasks. ICA architecture I produce spatially localized features which are only affected on some parts of the input image. It shows dominance in recognition system where the ICA architecture I produce better object recognition rather than PCA and ICA architecture II. In this paper, the author used ICA architecture I (which convert the input image to row vector for instance, $\mathrm{N} * \mathrm{~N}$ image convert to vector $\left.1 * \mathrm{~N}^{2}\right)$.

\section{PROPOSED METHOD}

The proposed system mainly consists of two parts, which are preprocessing and image recognition. Figure 1 shows the overall system that is used in this article and refers to the system performance as well.

\subsection{Enrolment}

By using local dataset images with extension .jpg for face recognition system, the local dataset have more than 40 students' images and each student has 8 images (Neutral, Smiling, Open Mouth, dim, bright, scarf, sunglass, scarf + sunglass). The datasets have been divided into two groups: training folder and testing folder. The training folder has 3 images (Neutral, Smiling, and Open mouth), and the testing folder contains other tested images (Dim light, Bright light, Sunglass, Scarf, and Sunglass + Scarf images). The local dataset has more than 360 images. The next figure shows a sample of student's images.

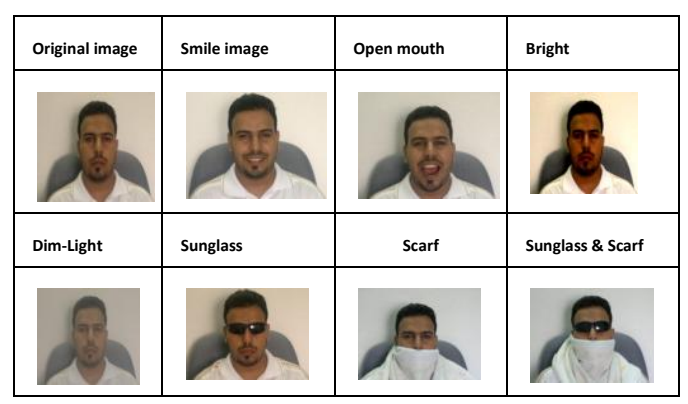

Figure 2. Shows a Sample of Student's Images

\subsection{Preprocessing}

The purpose of preprocessing is to remove artifacts from the dataset images. Preprocessing in this article has been done by implementing the following:

1-Feature Cartesian coordinates: marked using face recognition by Independent Component Analysis and the program is implemented using MATLAB. For each image mark, the eyes and mouth coordinate ( 1 person at a time, to avoid confusing names), the result from this program will be saved in a text file. Table 1 shows an example of the output.

Table 1. Shows Part of the Output Data from Step 1

\begin{tabular}{|l|c|c|c|c|c|c|}
\hline \multicolumn{1}{|c|}{$\begin{array}{c}\text { Image } \\
\text { name }\end{array}$} & \multicolumn{2}{c|}{$\begin{array}{c}\text { Right eye } \\
\text { X,Y position }\end{array}$} & \multicolumn{2}{c|}{$\begin{array}{c}\text { Left eye X, y } \\
\text { position }\end{array}$} & \multicolumn{2}{c|}{$\begin{array}{c}\text { Mouth X, y } \\
\text { position }\end{array}$} \\
\hline Dim & 199 & 277 & 284 & 245 & 245 & 368 \\
\hline Normal & 191 & 286 & 276 & 286 & 241 & 372 \\
\hline $\begin{array}{l}\text { Open } \\
\text { mouth }\end{array}$ & 192 & 280 & 279 & 282 & 239 & 366 \\
\hline Light & 189 & 273 & 280 & 275 & 241 & 361 \\
\hline Scarf & 206 & 282 & 292 & 290 & 248 & 385 \\
\hline Smile & 195 & 273 & 283 & 277 & 242 & 359 \\
\hline Sunglass & 207 & 273 & 288 & 278 & 247 & 355 \\
\hline $\begin{array}{l}\text { Sunglass_s } \\
\text { carf }\end{array}$ & 193 & 288 & 284 & 290 & 238 & 396 \\
\hline
\end{tabular}


2-Image Conversion and saving: Then, we use the csuFaceIdEval_5.1 [15], which uses the ANSII C code under Linux and does the following:

a- Extention conversion: The extension of images should be converted from .jpg into .pgm by using the com-mand: 1s *.jpg | awk -F. '\{ system("convert " \$0 " " \$1 ".pgm")\}'

We should make sure that the current directory is in the picture file which needs to be converting into .pgm.

b-Makefile compilation: Compile the makefile in the file"IluserlcsuFaceIdEval_5.1" by type make (e.g.: \$ make), and we will see an execution file appear in IluserlcsuFaceIdEval_5.1 bin $\mid x 86 \_64$. c- Running: Run the file name (csuPreprocessNormalize) at user/csuFaceIdEval_5.1/bin/x86_64 type./csuPreprocessNormalize.

d- Execution file option: An option of the execution file will appear; choose the option ./csuPreprocessNormalize -pgm $<$ output directory $><$ coordinate.txt directory $><$ picture directory>.

Replace the <> with your own directory and run the command.

e- Testing:Check the output directory to see the normalized .pgm file.

f- File opening:Finally, open the .pgm file in the MatLab.

\begin{tabular}{|l|l|l|l|l|l|l|l|l|}
\hline Original image & Normal & Smile & Open mouth & Bright & Dim-Light & Sunglass & Scarf & $\begin{array}{l}\text { Sunglass } \\
\text { with Scarf }\end{array}$ \\
\hline & & & & & & \\
\hline
\end{tabular}

Figure 3. Shows the Images after Preprocessing

\subsection{Image Recognition}

A template matching problem considered the simplest approach of image recognition. Problems arise when performing recognition in a high-dimensional space. Significant improvements can be achieved by first mapping the data into a lower-dimensional space. Figure 3 explains proposed method. The lower-dimensional space found using the idea behind eigenface is as follows[16]:

- Suppose $\Gamma$ is an $\mathrm{N} 2 \mathrm{x} 1$ vector, corresponding to an $\mathrm{NxN}$ face image I.

- The idea is to represent $\Gamma(\Phi=\Gamma$ - mean face $)$ in a lowdimensional space:

$$
\Phi^{\wedge}-\text { mean }=\mathrm{w} 1 \mathrm{u} 1+\mathrm{w} 2 \mathrm{u} 2+\ldots \mathrm{wK} \text { uK }(\mathrm{K}<<\mathrm{N} 2)
$$

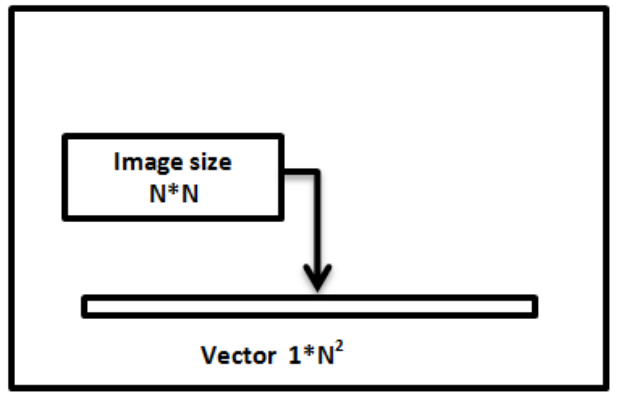

Figure 4 explains the proposed method (Arch1)

The computation of eigenfaces is as follows [4]:

Step 1: Obtain face images I1, I2, ..., IM (training faces) the face images should be centered and of the same size), as in Figure 5.

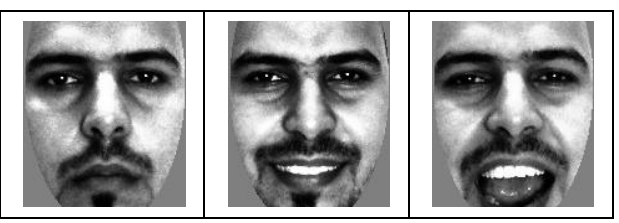

Figure 5. The training faces

Step 2: Represent every image Ii as a vector $\Gamma \mathrm{i}$

Step 3: Compute the average face vector $\Psi$ :

$$
\Psi=\frac{1}{M} \sum_{i=1}^{M} \Gamma i
$$

Step 4: Subtract the mean face:

$$
\Phi \mathrm{i}=\Gamma \mathrm{i}-\Psi
$$

Step 5: Compute the covariance matrix C:

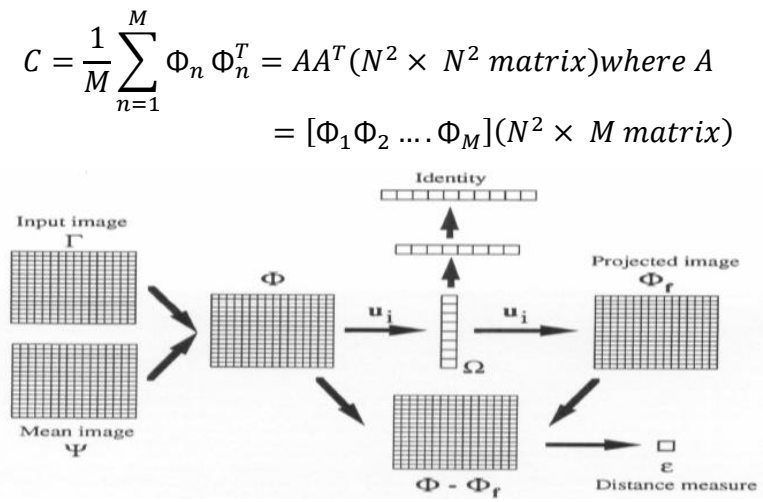

Figure 7 Shows how the ICA Algorithm Works 
The results of implementing Eigenface are shown in the next Figure.

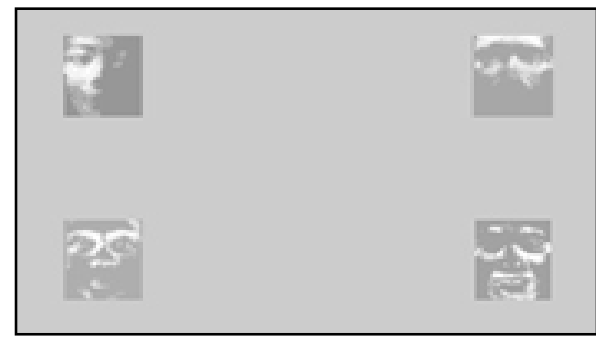

Figure 8. Shows the results of Eigenface

\section{EXPERIMENTAL RESULTS AND DISCUSSION}

In this paper, The ICA algorithm implemented using MATLAB R2012b and it tested on Dell INSPIRON PC Core 2Duo 2.20Ghz CPU with 4GB of RAM. The author used a dataset that has more than 40 students, and each student has 8 images (natural, bright, dim-light, smile, open mouth, sunglass, scarf, and scarf\&sunglass). The total time required to execute the program is about 30 seconds.

The results of proposed algorithm (ICA) are represented by using Cumulative Match Characteristic (CMC), the CMC shows the curve of plots of the probability between 1:M, where $\mathrm{M}$ is the number of people (Student) in the dataset. Figures(from 9 to 15) show the implementation of Arch1 for face recognition using the ICA algorithm on different images such as normal, bright, dim-light, smile, open mouth, sunglass, scarf, and scarf+sunglass. The proposed system find that the results of illumination images are better than those of the occlusion images (see Figures 14 and 15).

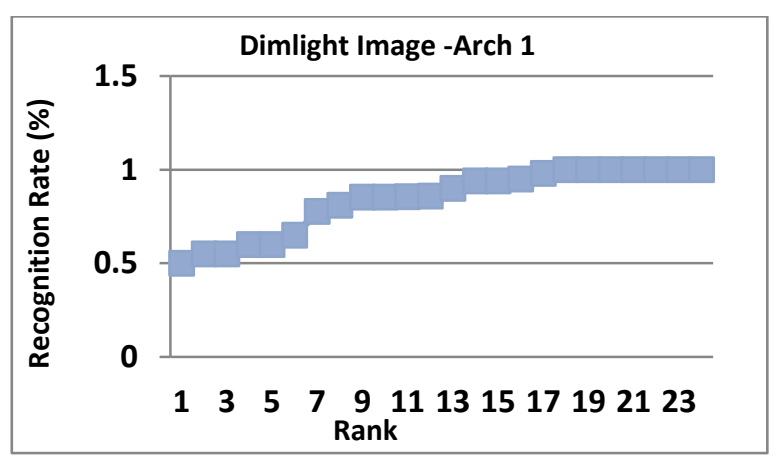

Figure 9. Dimlight Image Result using ICA

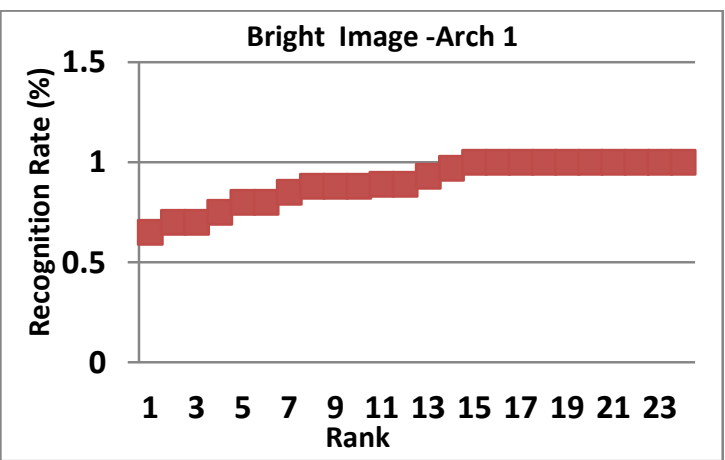

Figure 10.Bright Image Result using ICA

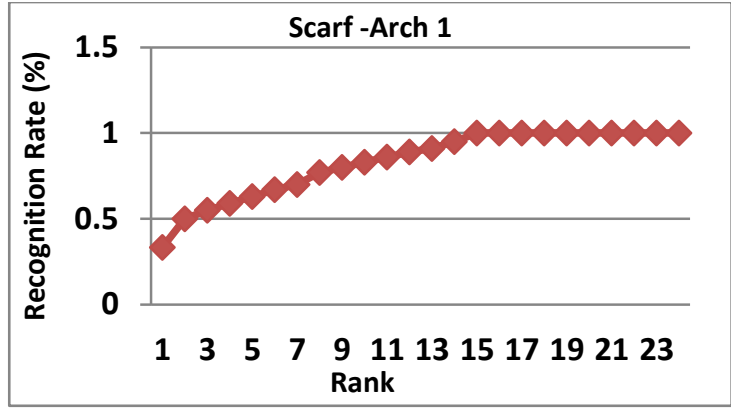

Figure 11.Scarf Image Result using ICA

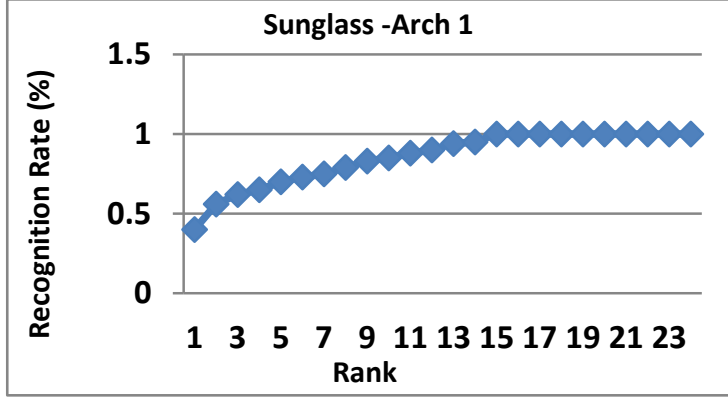

Figure 12.Sunglass Image Result using ICA

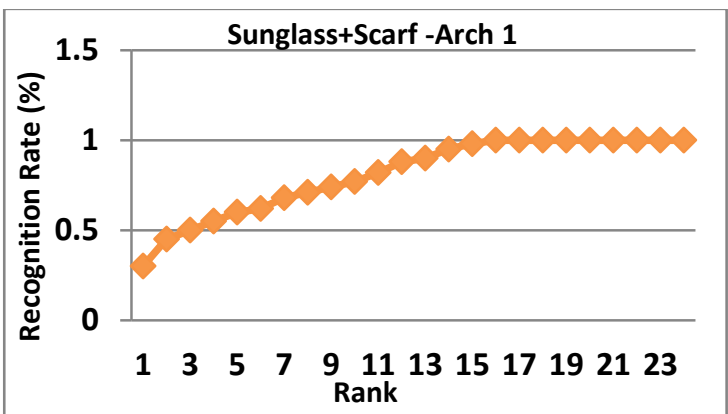

Figure 13.Sunglass and Scarf Image Result using ICA

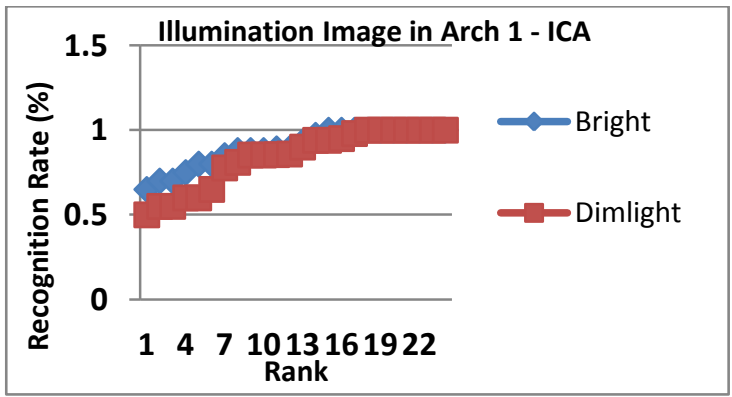

Figure 14.Illumination Image Result using ICA

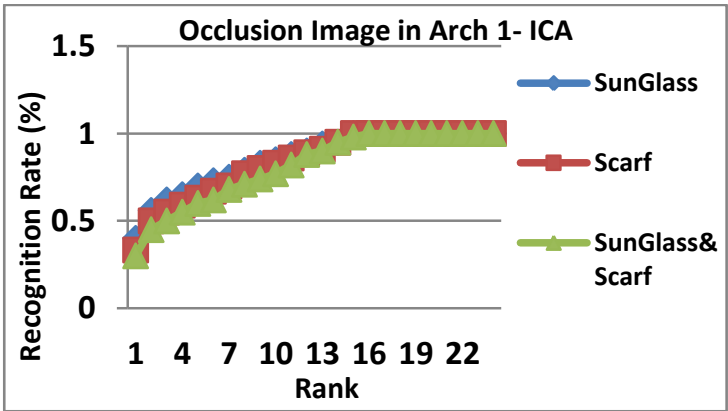

Figure 15.Occlusion Image Result using ICA 


\section{CONCLUSION AND FUTURE WORK}

In this paper, the author was implemented a face recognition system based on the ICA algorithm by using Matlab R2012b. The researcher used a local dataset that has more than 40 students' images, and each student has 8 images (Neutral, Smiling, Open Mouth, dim, bright, scarf, sunglass, scarf + sunglass). The data sets have been divided into two groups: training folder and testing folder. The training folder has 3 images (Neutral, Smiling, and Open mouth), and the testing folder contains five subfolders (Dim light images, Bright light images, Sunglass images, Scarf images, and Sunglass + Scarf images). The best results are found in illumination images (Dim light images, Bright light images). There is some limitation in the current system; this limitation has occurred as a result of the presence of some low-lying resolution images or the images containing noise; so, the solution for this problem is need to do some enhancement as a postpreprocessing. Figure 16 shows some of these images.

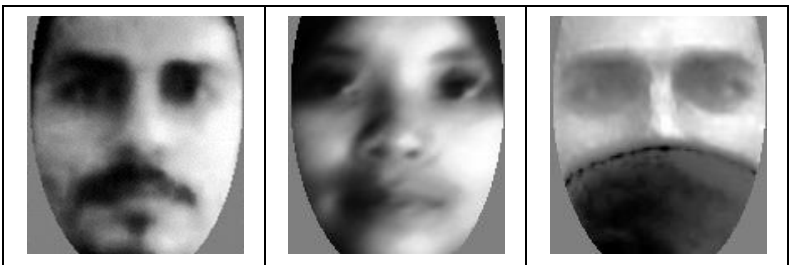

Figure 16: Shows some low-resolution images

For future work, we are looking at reducing the current run time; the author suggest using some image enhancement methods to remove blurring and noise from the original images such as by using the harmony search algorithm for image enhancement $[17,18]$.

\section{ACKNOWLEDGMENTS}

The author would like to thank Dr. Ibrahim Venkat and all the postgraduate students during 2012/2013 from the School of Computer Science / University Science Malaysia for supporting their work.

\section{REFERENCES}

[1] Ebtesam N. AlShemmary, "Fingerprint Image Enhancement and Recognition Algorithms", PhD. Thesis, University of Technology, Baghdad, 24 June, 2007.

[2] W. Zhao, R. Chellappa, A. Rosenfeld, P.J. Phillips, Face Recognition: A Literature Survey, ACM Computing Surveys, 2003, pp. 399-458

[3] Smith, Kelly. "Face recognition." PDF). Retrieved (2008): 06-04.

[4] P. S. Penev and J. J. Atick, "Local Feature Analysis: A General Statistical Theory for Object Representation”, Network: Comput. Neural Syst., vol. 7, no. 3, pp. 477500, 1996

[5] M. Turk and A. Pentland, "Eigenfaces for Recognition", J. CognitiveNeurosci., vol. 3, no. 1, pp. 71-86, 1991

[6] G. Cottrell and J. Metcalfe, "Face, Gender and Emotion Recognition Using Holons", in Advances in Neural Information Processing Systems, D. Touretzky, Ed. San
Mateo, CA: Morgan Kaufmann, 1991, vol. 3, pp. 564 571.

[7] P. Comon, "Independent Component Analysis-A New Concept”, Signal Processing, vol. 36, pp. 287-314, 1994.

[8] Matthew A. Turk and Alex P. Pentland, "Face Recognition Using Eigenfaces", $\quad$ CH29835/91/0000/0586 IEEE, 1991.

[9] Hyvärinen, Aapo, Juha Karhunen, and Erkki Oja. Independent component analysis. Vol. 46. John Wiley \& Sons, 2004.

[10] Rui Huang, Vladimir Pavlovic, and Dimitris N. Metaxas, "A Hybrid Face Recognition Method Using Markov Random Fields", ICPR (3) , pp. 157-160, 2004.

[11] A. J. Bell and T. J. Sejnowski, “An InformationMaximization Approach to Blind Separation and Blind Deconvolution", Neural Comput., vol. 7, no. 6, pp. 1129-1159, 1995.

[12] Bell, Anthony J., and Terrence J. Sejnowski, "The Independent Components of Natural Scenes are Edge Filters", Vision Res., vol. 37, no. 23, pp. 3327-3338, 1997.

[13] M. S. Bartlett, "Face Image Analysis by Unsupervised Learning: Kluwer Academic", 2001.

[14] M. S. Bartlett and T. J. Sejnowski, "Viewpoint Invariant Face Recognition Using Independent Component Analysis and Attractor Networks", in Neural Information Processing Systems - Natural and Synthetic, M. Mozer, M. Jordan, and T. Petsche, Eds. Cambridge, MA: MIT Press, vol. 9, pp. 817-823,1997.

[15] Beveridge, J.R., et al., "The CSU Face Identification Evaluation System", Machine Vision and Applications, 16(2), p.p 128-138, 2005.

[16] M. Turk and A. Pentland, "Eigenfaces for Recognition", Journal of Cognitive Neuroscience, vol.3, no. 1 , pp. $71-86,1991$.

[17] Peter T. Higgins, "Introduction to Biometrics", http://www.thehhg.com Sep., 2005

[18] Zaid Abdi Alkareem, Y. A., et al. "Edge preserving image enhancement via harmony search algorithm." Data Mining and Optimization (DMO), 2012 4th Conference on. IEEE, 2012

\section{AUTHORS PROFILE}

Zaid Abdi Alkareem Alyasseri : (b. 1985 Najaf/Iraq) graduated with a BSc in computer science from Babylon University in 2007 and received MSc in computer science from University Science Malaysia (USM) in 2013. Author works as an Asst. Lecturer in Electronics and Communication Engineering Dept. at the University of Kufa-Iraq. He has experience for 10 years in software development and system analysis also he has good experience in many programming languages and database such as : $\mathrm{C}++$, VB.net, Java, Matlab, SQL, MySQL, Oracle, Python. Authors' research interests are computer vision and image processing, computer graphics processing, programming, and parallel computing. 